\title{
The Current State of Radiopharmaceutical Therapy
}

\author{
Chaitanya Divgi \\ Divgi Consulting LLC, Meadowbrook, Pennsylvania
}

See the associated article on page 1708.

$\mathbf{M}$

any of us believe the roots of nuclear medicine are as much therapeutic as diagnostic. In a scenario mirroring the current theranostic paradigm, the first nuclear medicine radionuclide, ${ }^{131} \mathrm{I}$, was explored for its ability to interrogate the pathophysiologic features of thyroid disorders at about the same time as it was explored for its therapeutic utility.

${ }^{131}$ I therapy, however, remains both an art and a standardized procedure. Therapy with ${ }^{131} \mathrm{I}$ —not only for hyperthyroidism but for thyroid cancer-varies across institutions, with consideration being paid to logistic issues and convenience in addition to possible adverse effects and efficacy (1). This variation may be a function of both the large therapeutic window and the relatively benign natural course of these diseases.

Increasing cost constraints and the consequent reluctance of institutional payers now necessitates greater rigor and standardization. Radiopharmaceutical therapy in cancer needs approval in a manner comparable to that for any other oncologic therapy, with hard measures of efficacy and strict regimens of therapy that specify radioactivity amounts and administration schedules.

Randomized phase III trials are thus perhaps a prerequisite for Food and Drug Administration approval. Approval of ${ }^{153} \mathrm{Sm}-$ ethylenediamine tetra(methylene phosphonic acid) $\left({ }^{153} \mathrm{Sm}\right.$-lexidronam [Quadramet; Lantheus]) and ${ }^{223}$ Ra-dichloride (Xofigo; Bayer) followed rigorous demonstration of subjective (pain control) and objective (survival) improvement, respectively, compared with a statistically meaningful control population $(2,3)$.

Comparable rigor in the therapy trial of gastroenteropancreatic neuroendocrine tumors using a ${ }^{177} \mathrm{Lu}$-labeled peptide $\left({ }^{177} \mathrm{Lu}-\right.$ DOTATATE [Lutathera; Advanced Accelerator Applications]) targeting a somatostatin receptor subtype (4) resulted in priority review of the agent by the Food and Drug Administration. The Food and Drug Administration was responsive to health-care needs and facilitated an expanded-access program to gather more efficacy data that enabled agent approval (5).

For several decades, neuroendocrine tumors that overexpress norepinephrine transporter have been treated with ${ }^{131} \mathrm{I}$ labeled to a norepinephrine analog, benzylguanidine. The resultant compound- ${ }^{131}$ I-meta-iodo-benzylguanidine (or ${ }^{131} \mathrm{I}-\mathrm{MIBG}$ )— has been

Received Jun. 11, 2018; revision accepted Jun. 23, 2018.

For correspondence or reprints contact: Chaitanya Divgi, Divgi Consulting

LLC, 1417 Robinhood Rd., Meadowbrook, PA 19046.

E-mail: crdivgi@gmail.com

Published online Jun. 29, 2018.

COPYRIGHT @ 2018 by the Society of Nuclear Medicine and Molecular Imaging. DOI: 10.2967/jnumed.118.214122 used outside the United States, much like radioiodine therapy, in a schedule determined both by efficacy and by logistics ( 6 ). Efforts to enhance the availability and use of this therapy were perhaps inevitable given its demonstrated efficacy in a theranostic setting, exemplified by imaging to confirm biodistribution and tumor targeting followed by therapy with a large amount of radioactivity. Edward Coleman at Duke University led the systematic evaluation of ${ }^{131} \mathrm{I}$ MIBG therapy in adult NET (7), and an article by Kane et al. in this issue of The Journal of Nuclear Medicine (8) continues that fine tradition.

The retrospective review by Kane et al. demonstrated significant symptom relief and survival benefit in 211 patients treated with ${ }^{131}$ I-MIBG for metastatic neuroendocrine tumors at Duke University Hospital from 1991 to 2014 (8). The review confirms what the community has known for quite a while: ${ }^{131}$ I-MIBG therapy results in symptom improvement, and a delay in tumor growth, in a substantial proportion of treated patients. (Improvement over existing therapy could only be suggested in this retrospective review without a matched control.)

The article also points out the need for continuing rigor in the clinical testing of all radiopharmaceutical therapies: "In particular, future studies would benefit from more consistent documentation of performance status in the clinical record, as well as the use of some objective index of general wellness to separate the influence of multiple therapies independent of the propensity to survive." There are several such indices, and their application is routine in most oncology practice (9).

The authors end their Discussion by pointing out the weaknesses-largely involving missing data regarding patient statusthat preclude greater confidence in the conclusions of their study. That experience should act as a cautionary tale that guides our clinical conduct; we need to adopt the highest oncology practice standards to evaluate our radiopharmaceutical therapies and enable them to be approved, accepted, and used as an integral option for an increasing number of diseases. We need to become as facile at recording patient performance scores and hematopoietic indices as we are at recording administered radioactivity amounts and absorbed doses.

Nuclear medicine incorporates chemistry, physics, biology, and instrumentation, among other broad fields of knowledge. Clinical nuclear medicine needs to incorporate other imaging as well as therapeutic modalities to optimize use of the manifold ways in which this exciting discipline affects health care, particularly in cancer. Our discipline is not a part of some other but rather is a whole that is greater than the sum of its myriad parts (themselves components of other specialties and scientific fields).

As nuclear medicine physicians, we have 2 broad clinical responsibilities. We evaluate images of in vivo radiopharmaceutical distribution to make clinical decisions, and we treat patients with therapeutic radiopharmaceuticals, bringing to bear our understanding of (molecular and clinical) radiochemistry, biology, and 
pathophysiology. Our unique molecular imaging capabilities need to be combined with morphologic imaging (conventional imaging) to provide insight into pathophysiologic processes; therefore, we need to be well versed in all aspects of in vivo imaging. Only thus are we able to gain the confidence of referring clinician and patient alike.

We must similarly be well versed in all aspects of radiopharmaceutical therapy, not just those that relate to radiation safety and toxicity. We need to take care of our patients, not only administering the radiopharmaceutical but also considering ways to ameliorate toxicity or enhance efficacy with other treatment modalities. We need to work closely with our patients as well as with our clinical colleagues. We need to be clinicians, expert in all aspects of patient care and rigorously attending to clinical practice and management. The only way we can optimize use of our therapeutic arsenal is by becoming accepted as clinicians by our clinical colleagues.

\section{DISCLOSURE}

No potential conflict of interest relevant to this article was reported.

\section{REFERENCES}

1. Silberstein EB, Alavi A, Balon HR, et al. The SNMMI practice guideline for therapy of thyroid disease with ${ }^{131}$ I 3.0. J Nucl Med. 2012;53:1633-1651.

2. Serafini AN, Houston SJ, Resche I, et al. Palliation of pain associated with metastatic bone cancer using samarium-153 lexidronam: a double-blind placebo-controlled clinical trial. J Clin Oncol. 1998;16:1574-1581.

3. Parker C, Nilsson S, Heinrich D, et al;; ALSYMPCA investigators. Alpha emitter radium-223 and survival in metastatic prostate cancer. $N$ Engl J Med. 2013;369:213-223.

4. Strosberg J, El-Haddad G, Wolin E, et al.; NETTER-1 trial investigators. Phase 3 trial of ${ }^{177} \mathrm{Lu}$-Dotatate for midgut neuroendocrine tumors. $N$ Engl J Med. 2017; 376:125-135.

5. FDA approves new treatment for certain digestive tract cancers. U.S. Food and Drug Administration website. https://www.fda.gov/NewsEvents/Newsroom/PressAnnouncements/ ucm594043.htm. Published January 26, 2018. Accessed August 2, 2018.

6. Giammarile F, Chiti A, Lassmann M, et al. EANM procedure guidelines for ${ }^{131} \mathrm{I}-$ meta-iodobenzylguanidine $\left.{ }^{131} \mathrm{I}-\mathrm{mIBG}\right)$ therapy. Eur J Nucl Med Mol Imaging. 2008;35:1039-1047.

7. Coleman RE, Stubbs JB, Barrett JA, de la Guardia M, Lafrance N, Babich JW. Radiation dosimetry, pharmacokinetics, and safety of ultratrace iobenguane I-131 in patients with malignant pheochromocytoma/paraganglioma or metastatic carcinoid. Cancer Biother Radiopharm. 2009;24:469-475.

8. Kane A, Thorpe MP, Morse MA, et al. Predictors of survival in 211 patients with stage IV pulmonary and gastroenteropancreatic MIBG-positive neuroendocrine tumors treated with ${ }^{131}$ I-MIBG. J Nucl Med. 2018;59:1708-1713.

9. Oken MM, Creech RH, Tormey DC, et al. Toxicity and response criteria of the Eastern Cooperative Oncology Group. Am J Clin Oncol. 1982;5:649-655. 